\title{
Substrate-Independent Growth of Atomically Precise Chiral Graphene Nanoribbons
}

Dimas G. de Oteyza, ${ }^{* \dagger, \uparrow, \S}$ Aran García-Lekue, ${ }^{\dagger \dagger}$ Manuel Vilas-Varela, " Néstor Merino-Díez, ${ }^{\dagger, \perp}$ Eduard Carbonell-Sanromà, ${ }^{\perp}$ Martina Corso, ${ }^{\ddagger},{ }^{\perp} \perp$ Guillaume Vasseur, ${ }^{\dagger, \S}$ Celia Rogero, ${ }^{\dagger, \S}$ Enrique Guitián, " Jose Ignacio Pascual, ${ }^{\ddagger} \perp$ J. Enrique Ortega, ${ }^{\dagger, \S, \# ~ Y u t a k a ~ W a k a y a m a, ~}{ }^{\mathbb{T}}$ and Diego Peña*, ${ }^{*}$

${ }^{\dagger}$ Donostia International Physics Center (DIPC), Paseo Manuel Lardizabal 4, 20018 San Sebastián, Spain

${ }^{\ddagger}$ Ikerbasque, Basque Foundation for Science, 48011 Bilbao, Spain

${ }^{\S}$ Materials Physics Center, Centro de Física de Materiales (CSIC/UPV-EHU), Paseo Manuel Lardizabal 5, 20018 San Sebastián, Spain

"Centro de Investigación en Química Biolóxica e Materiais Moleculares (CIQUS) and Departamento de Química Orgánica, Universidade de Santiago de Compostela, 15782 Santiago de Compostela, Spain

${ }^{\perp}$ CIC nanoGUNE, Avenida de Tolosa 76, 20018 San Sebastián, Spain

\#Departamento de Física Aplicada I, Universidad del País Vasco, 20018 San Sebastián, Spain

II International Center of Materials Nanoarchitectonics, National Institute for Materials Science, 1-1 Namiki, Tsukuba 305-0044, Japan

Supporting Information

ABSTRACT: Contributing to the need for new graphene nanoribbon (GNR) structures that can be synthesized with atomic precision, we have designed a reactant that renders chiral $(3,1)$-GNRs after a multistep reaction including Ullmann coupling and cyclodehydrogenation. The nanoribbon synthesis has been successfully proven on different coinage metals, and the formation process, together with the fingerprints associated with each reaction step, has been studied by combining scanning tunneling microscopy, core-level spectroscopy, and density functional calculations. In addition to the GNR's chiral edge structure, the substantial GNR lengths achieved and the low processing temperature required to complete the reaction grant this reactant extremely interesting properties for potential applications.

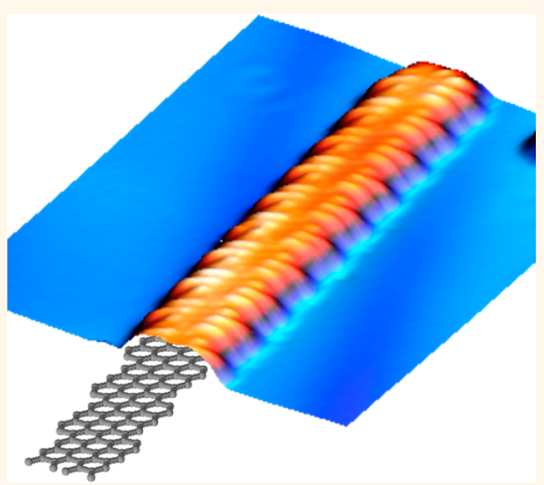

KEYWORDS: on-surface synthesis, Ullmann coupling, cyclodehydrogenation, scanning tunneling microscopy, core-level photoemission, density functional theory

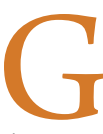
raphene nanoribbons (GNRs) are drawing enormous interest, partly due to their attractive electronic properties. ${ }^{1,2}$ Those properties vary dramatically with changes in the nanoribbon's atomic structure in terms of width, ${ }^{3-5}$ crystallographic symmetry, ${ }^{6,7}$ dopant heteroatoms, ${ }^{8-13}$ and edge termination. ${ }^{14}$ Moreover, the electronic properties can be modulated even further by the appropriate design of GNR heterostructures. ${ }^{12,15-17}$ This enormous tunability of electronic properties is thus extremely promising for next-generation nanoelectronic and optoelectronic devices. ${ }^{18,19}$ However, the high susceptibility of those properties to minimum changes in the GNR structure also indicates the stringent need for atomic precision in GNR synthesis. With the advent of bottom-up synthesis, ${ }^{1,2,20}$ increasingly high hopes are being placed on this approach, but the field is still in its infancy. Thus, although a large pool of GNRs with different edge orientations, widths, or heteroatoms (and heterostructures thereof) should become available to really allow for the envisioned breakthroughs in nanoelectronics and the development of full GNR-based circuitry; so far, only few GNRs have been successfully synthesized with the required selectivity and precision. ${ }^{1,2,8-10,20-24}$

To date, the most widely studied nanoribbon is the armchairoriented GNR with seven dimer lines across its width (7AGNR) that grows from $10,10^{\prime}$-dibromo-9,9'-bianthracene (reactant $\mathbf{1}$ in Figure 1a) in a multistep reaction, including dehalogenation, polymerization (also known as Ullmann coupling), and cyclodehydrogenation..$^{20,25,26}$ The synthesis of 7-AGNR has been shown to work reproducibly on substrates such as $\mathrm{Au}(111),{ }^{20} \mathrm{Au}(110),{ }^{27}$ or $\mathrm{Ag}(111) .{ }^{20,28}$ Surprisingly,

Received: August 4, 2016

Accepted: August 22, 2016

Published: August 22, 2016 


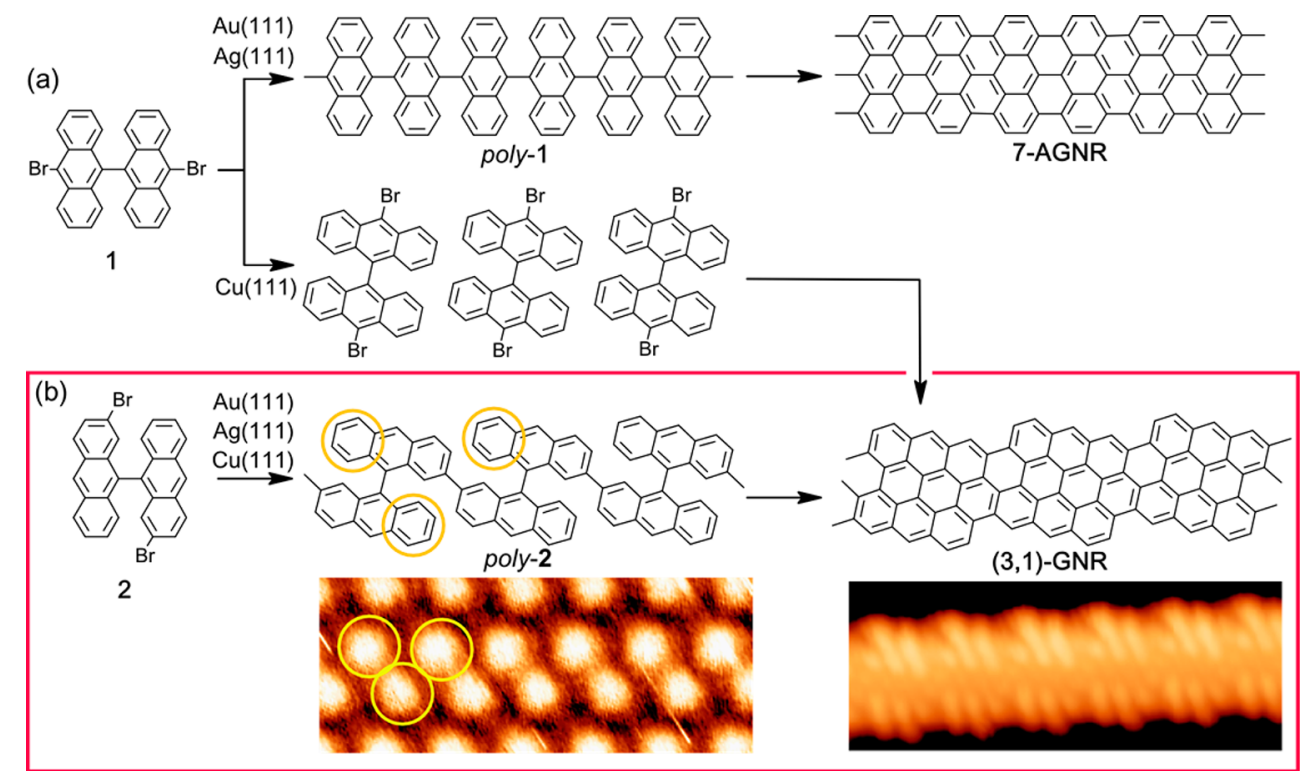

Figure 1. (a) Schemes of the chemical reactions of precursor 1 on various metallic surfaces. $\mathrm{On} \operatorname{Au}(111)$ and $\mathrm{Ag}(111)$, it affords armchair GNRs. On $\mathrm{Cu}(111)$, it affords chiral $(3,1)$-GNRs. (b) Our work (highlighted with the red line) reports the transformation of reactant 2 into chiral GNRs independently of the substrate $[\mathrm{Au}(111), \operatorname{Ag}(111)$, and $\mathrm{Cu}(111)]$. Associated STM images are shown for poly-2 after initial polymerization by Ullmann coupling $(5.6 \mathrm{~nm} \times 2.2 \mathrm{~nm}, I=0.09 \mathrm{nA}, U=1.5 \mathrm{~V})$, as well as for the final $(3,1)$-GNR after cyclodehydrogenation $(5.6 \mathrm{~nm} \times 2.2 \mathrm{~nm}, I=0.2 \mathrm{nA}, U=-650 \mathrm{mV})$, both on $\mathrm{Au}(111)$. Steric hindrance causes poly-2 to be nonplanar. The high parts (circled in yellow) are correspondingly marked in the polymer's wireframe structure above.

the same reactant 1 designed to render AGNRs turned out to form chiral (3,1)-GNRs on $\mathrm{Cu}(111)$ (Figure 1). ${ }^{29,30}$ This result has been subject to debate ${ }^{31-34}$ since the polymerization does not involve the carbon atoms attached to bromines. However, the debate has been recently settled by unambiguous highresolution imaging of the resulting bonding structure. ${ }^{35}$ These results mirror a very system-specific reaction mechanism not translatable to other substrates, based on the surface-catalyzed, selective activation of particular $\mathrm{C}-\mathrm{H}$ bonds. In fact, similar results were obtained from $\mathbf{1}$ and its nonhalogenated sister molecule, the latter producing slightly longer GNRs. ${ }^{35}$ This implies an absent or even negative impact of the halogenation of 1 for GNR growth on $\mathrm{Cu}(111)$.

Nevertheless, those results have inspired this work, in which we introduce monomer 2 , specifically designed to obtain $(3,1)$ GNRs in a more efficient manner (Figure 1). In particular, the bromine atoms in positions $10,10^{\prime}$ of reactant 1 have been shifted in reactant 2 to positions $2,2^{\prime}$, which are the positions selectively activated on $\mathrm{Cu}(111)$ and through which the polymerization preceding the $(3,1)$-GNR formation takes place. The same structure can thus be obtained from 2 following a conventional Ullmann coupling/cyclodehydrogenation sequence. This has been shown to work independently of the used substrate, as proven with growth studies on $\mathrm{Au}(111)$ and $\mathrm{Ag}(111)$. A similar study on $\mathrm{Cu}(111)$ is out of the scope of this paper due to the readily proven growth of $(3,1)$-GNRs from 1 (and even from nonhalogenated precursors) on that substrate. However, also on $\mathrm{Cu}(111)$, we show how changing the halogen functionalization site, and thereby changing the polymerization mechanism from a selective $\mathrm{C}-\mathrm{H}$ bond activation to Ullmann coupling, is still a significant advancement by greatly increasing the resultant GNR length.

\section{RESULTS AND DISCUSSION}

Key to this work is the synthesis of bianthracene reactants with adequately chosen bromine atom positions. We prepare $2,2^{\prime}$ dibromo-9, $9^{\prime}$-bianthracene (2) starting from phthalic anhydride (3), following the four-step synthetic route shown in Figure 2 (see Supporting Information, Scheme S1 and Figures S1 and S2 for details). The key reaction in this protocol is the reductive coupling of bromoanthrone 4 promoted by $\mathrm{Zn} .^{36}$

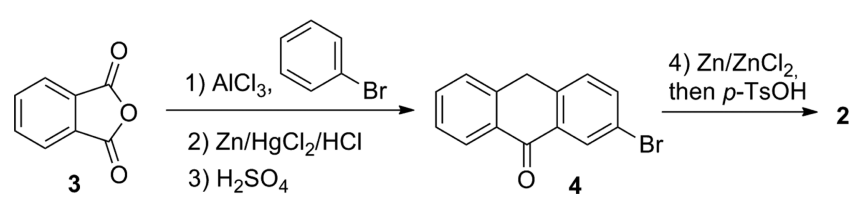

Figure 2. Synthetic route to obtain dibromobianthracene 2 from phthalic anhydride 3.

Starting from reactant 2 , the reaction pathway associated with the surface-supported synthesis of $(3,1)$-GNRs is closely related to the well-known transformation of 1 into 7-AGNRs. That is, in a first step, the molecules polymerize by Ullmann coupling into poly-2. This polymer is a highly nonplanar molecular structure in which the steric hindrance between the $\mathrm{H}$ atoms in neighboring anthracene units drives their alternating tilting (Figure $1 \mathrm{~b}$ ). As a consequence, the polymer's imaging by scanning tunneling microscopy (STM) displays a sequence of protruding features that we associate with the uppointing ends of the anthracene units. This correspondence is highlighted by yellow circles in the wireframe chemical structure and in the STM image. In a following reaction step, cyclodehydrogenation sets in and poly- 2 transforms into the planar $(3,1)$-GNR structure, as can be directly discerned in the high-resolution STM images in Figure $1 \mathrm{~b}$ and Figure S3.

Figure 3 summarizes, as observed by STM, the growth process of (3,1)-GNRs on top of $\mathrm{Au}(111)$ and $\mathrm{Ag}(111)$. On 

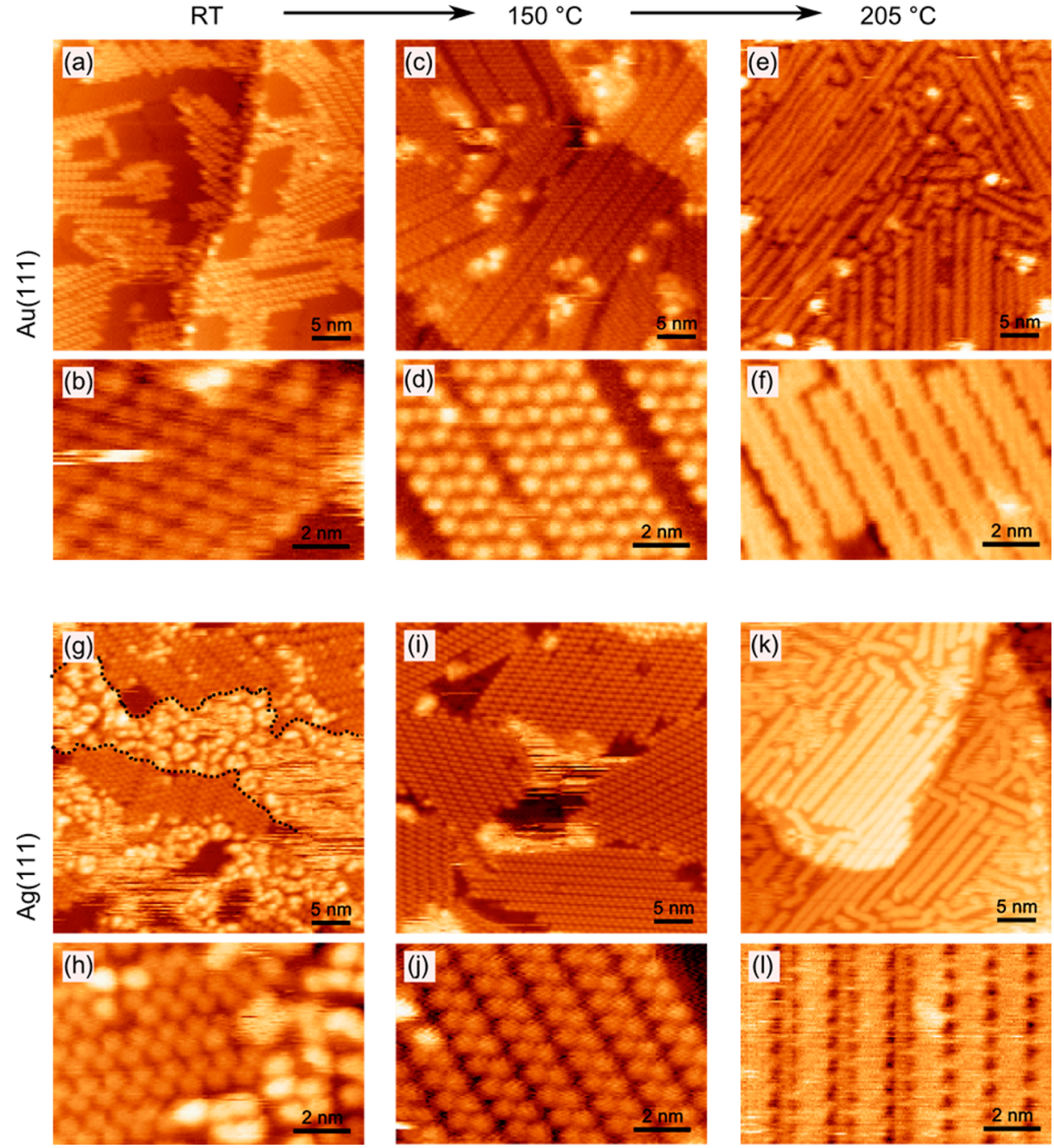

Figure 3. Large-scale $(36 \mathrm{~nm} \times 36 \mathrm{~nm})$ and smaller-scale $(10 \mathrm{~nm} \times 6.5 \mathrm{~nm})$ STM images on $\mathrm{Au}(111)(\mathrm{a}-\mathrm{f})$ and $\mathrm{Ag}(111)(\mathrm{g}-\mathrm{l})$ of the same samples at different growth stages: after deposition on substrates held at room temperature $(\mathrm{a}, \mathrm{b}, \mathrm{g}, \mathrm{h})$, after being annealed to $150{ }^{\circ} \mathrm{C}(\mathrm{c}, \mathrm{d}, \mathrm{i}, \mathrm{j})$, and after being annealed to $205^{\circ} \mathrm{C}(\mathrm{e}, \mathrm{f}, \mathrm{k}, \mathrm{l})$. STM imaging parameters are (a) $I=0.086 \mathrm{nA}, U=1.4 \mathrm{~V}$; (b) $I=0.086 \mathrm{nA}, U=1.5 \mathrm{~V}$; (c) $I=0.16$ $\mathrm{nA}, U=1.76 \mathrm{~V}$; (d) $I=0.16 \mathrm{nA}, U=1.76 \mathrm{~V}$; (e) $I=0.16 \mathrm{nA}, U=0.47 \mathrm{~V}$; (f) $I=1.29 \mathrm{nA}, U=-0.13 \mathrm{~V}$; (g) $I=0.09 \mathrm{nA}, U=1.5 \mathrm{~V}$; (h) $I=0.06$ $\mathrm{nA}, U=-2.02 \mathrm{~V}$; (i) $I=0.36 \mathrm{nA}, U=1.76 \mathrm{~V}$; (j) $I=0.36 \mathrm{nA}, U=-1.6 \mathrm{~V}$; (k) $I=0.42 \mathrm{nA}, U=1.07 \mathrm{~V}$; and $(\mathrm{l}) I=0.19 \mathrm{nA}, U=-0.13 \mathrm{~V}$.

either substrate, the images correspond to the same sample at different stages of its growth: after deposition of $\mathbf{2}$ on substrates held at room temperature, after being annealed to $150^{\circ} \mathrm{C}$, and after being annealed to $205{ }^{\circ} \mathrm{C}$.

We first focus on $\mathrm{Au}(111)$. After room temperature deposition, the molecules aggregate into islands of linear structures formed by a zig-zag arrangement of protrusions comparable to those expected from poly-2 (Figure 3a,b). Upon annealing to $150{ }^{\circ} \mathrm{C}$, we observe clear changes in the sample's topology. It is difficult to discern changes in the STM contrast within the linear structures (see Supporting Information for details). However, their overall length substantially increases and the spacing between them (perpendicular to the structure's long axis) becomes less regular and decreases the minimum distance (Figure 3c,d). Annealing to $205^{\circ} \mathrm{C}$ brings about more notorious changes, displaying arrays of planar structures clearly recognizable from the edge topology as $(3,1)$-GNRs (Figure $3 e, f)$.

In the case of $\operatorname{Ag}(111)$, two distinct sections are observed after molecular deposition at room temperature. On the one hand, we find regions of ordered, linear structures packed side by side (Figure $3 \mathrm{~g}$ ). The linear structures are imaged again as zig-zagging protrusions (Figure $3 \mathrm{~h}$ ). Instead, other regions display a disordered arrangement of adsorbates with increased mobility and a much larger apparent height $(\sim 2.7 \AA$ $v s \sim 1.8 \AA$ ) . The areal ratio between these two different sections is approximately 1:1 (Figure 3h). Annealing to $150{ }^{\circ} \mathrm{C}$ brings about the growth of the ordered, linear structures at the expense of the disappearing disordered regions (Figure 3i,j). As opposed to the findings on $\mathrm{Au}(111)$, on $\mathrm{Ag}(111)$, the arrangement within the ordered arrays of linear structures remains unchanged after this annealing. Annealing the sample to $205{ }^{\circ} \mathrm{C}$ triggers the cyclodehydrogenation and thereby the ultimate formation of $(3,1)$-GNRs (Figure 3k,1).

Complementary information on the chemical transformation process is obtained from core-level X-ray photoemission spectroscopy (XPS) measurements. As in the STM experiment, molecules were deposited on $\mathrm{Au}(111)$ and $\mathrm{Ag}(111)$ substrates held at room temperature. The samples were then annealed 

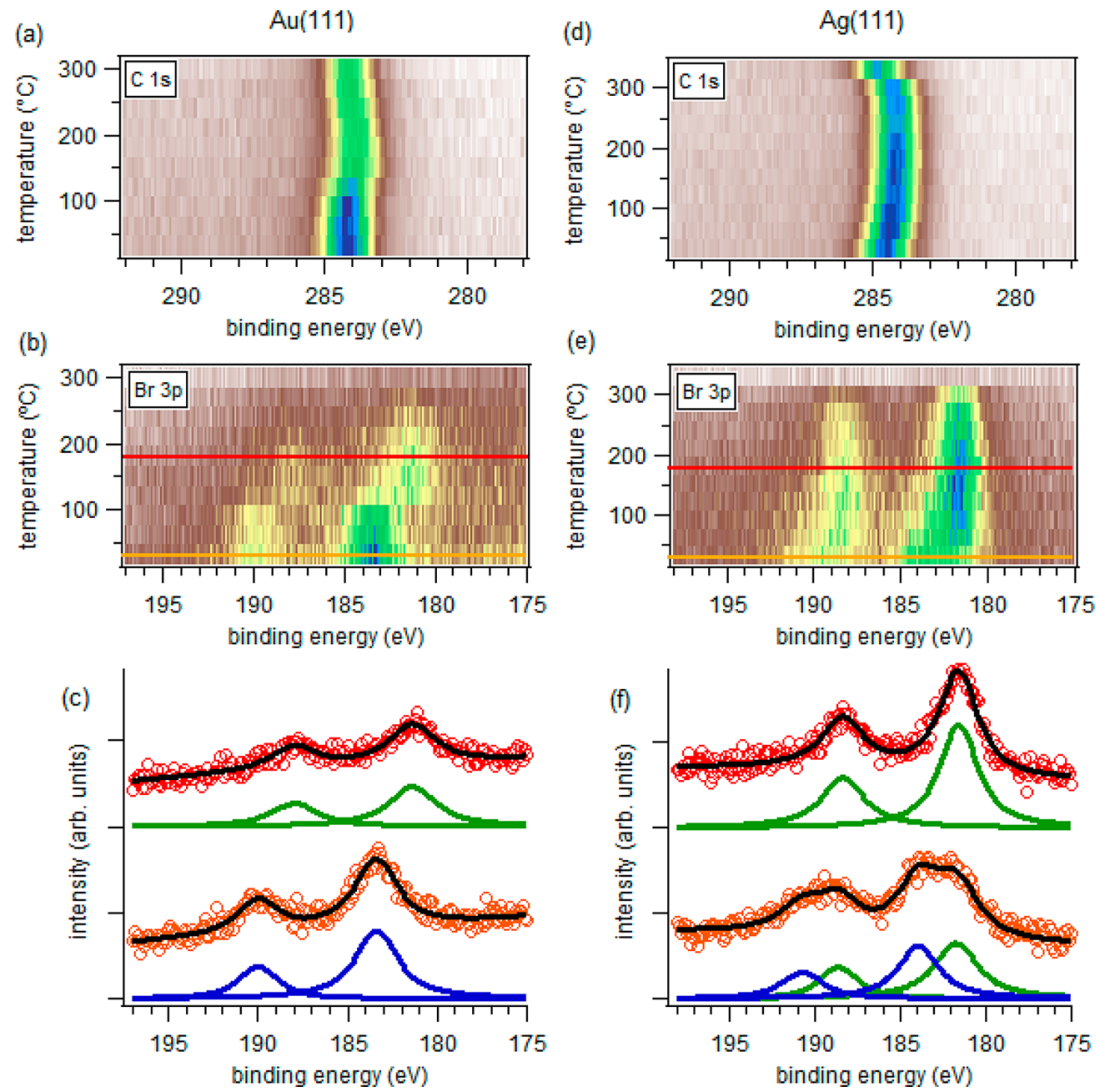

Figure 4. Photoemission spectra of the $\mathrm{C}$ 1s core levels of 2 deposited on (a) $\operatorname{Au}(111)$ and (d) $\operatorname{Ag}(111)$ held at room temperature and their evolution as a function of sample annealing temperature. Similar measurements of the $\mathrm{Br} 3 \mathrm{p}$ core levels are shown in panels (b) and (e). (c,f) $\mathrm{Br} 3 \mathrm{p}$ spectra, together with their associated fits (blue and green lines correspond to organic and metal-bound Br components, respectively), of two representative temperatures marked with the colored lines in (b) and (e), respectively. The spectra are shifted along the intensity axis for better comparison.

stepwise while their $\mathrm{Br} 3 \mathrm{p}$ and $\mathrm{C} 1$ s core-level spectra were monitored. The data are summarized in Figure 4.

$\mathrm{On} \mathrm{Au}(111)$, the molecules remain intact upon deposition at room temperature and only start showing chemical changes for substrate temperatures of around $125^{\circ} \mathrm{C}$. As the temperature increases above that threshold, the most evident change in the core-level spectra is a pronounced shift of the $\mathrm{Br} 3 \mathrm{p}$ peaks to $\sim 2 \mathrm{eV}$ lower binding energies. This effect is well-known from other studies on Ullmann coupling of different precursors and relates to the dehalogenation process and the new chemical environment as $\mathrm{Br}$ detaches from the organic molecule and binds to the metallic surface. ${ }^{27,37,38}$ Concomitantly, the $\mathrm{C} 1 \mathrm{~s}$ peak displays a smaller shift $(\sim 0.3 \mathrm{eV})$ to lower binding energies. Similar C 1s shifts have also been observed in previous studies on Ullmann coupling with different precursors and surfaces, for which a variety of explanations have been given: (i) bond formation between the $\mathrm{C}$ atoms hosting the generated radicals and the substrate atoms or adatoms, ${ }^{27,37}$ (ii) a change in the supramolecular assembly prior to dehalogenation that brings about changes in the interaction with the substrate, ${ }^{38}$ or (iii) a change of work function caused by the chemisorption of $\mathrm{Br}$ to the substrate. ${ }^{37} \mathrm{We}$ discard the first because on $\mathrm{Au}$ the formation of organometallic compounds is disfavored and the molecules are known to polymerize as the radicals are formed. ${ }^{39,40}$ We also discard the second because we observe the $\mathrm{C} 1 \mathrm{~s}$ and $\mathrm{Br} 3 \mathrm{p}$ shifts simultaneously in a correlated way. Thus, we ascribe the observed $\mathrm{C} 1 \mathrm{~s}$ shift to the change of work function generated by the metal-bound $\mathrm{Br}$ atoms. Besides, this is further supported by the changes observed in the core levels as the temperature is increased further: as $\mathrm{Br}$ desorption starts to set in, the $\mathrm{C} 1 \mathrm{~s}$ level shifts in the opposite direction, toward higher binding energy. A similar shift on closely related systems has also been ascribed to different reaction processes like the transformation from an organometallic to a polymer phase ${ }^{27,41}$ or cyclodehydrogenation. ${ }^{37}$ In addition to the chemical change, these reactions also cause an alteration in the moleculesubstrate distance that may, in turn, additionally affect the corehole screening effects and thereby the core-level spectra. However, we can again discard these justifications for our system because we know the polymerization and cyclodehydrogenation to occur at lower temperatures. Altogether, XPS on $\mathrm{Au}(111)$ thus shows that the structures observed on $\mathrm{Au}(111)$ at room temperature are a noncovalent self-assembled supramolecular arrangement, polymerizing only upon annealing above $125^{\circ} \mathrm{C}$, in line with the overall sample topology changes observed by STM at $150{ }^{\circ} \mathrm{C}$ and described above.

On $\mathrm{Ag}(111)$, the molecules are readily partially dehalogenated upon deposition at room temperature. This is clearly observed in the $\mathrm{Br} 3 \mathrm{p}$ core-level spectra, which shows the coexistence of organic and metal-linked $\mathrm{Br}$ atoms in a 1:1 ratio. As the temperature is increased, the metal-bound $\mathrm{Br}$ increases at the expense of the organic Br. From the correlation with the STM observations, we can readily ascribe the pristine precursors to the disordered structures found at room temperature and the dehalogenated molecules to be the building blocks forming the ordered structures. As in 
$\mathrm{Au}(111)$, the dehalogenation brings about a minor shift to lower binding energy in the $\mathrm{C} 1 \mathrm{~s}$ spectrum, which shifts back again as the $\mathrm{Br}$ is desorbed at higher temperatures. Again, we ascribe the dominating effect behind the $\mathrm{C} 1 \mathrm{~s}$ shifts to the changes in work function as $\mathrm{Br}$ binds or leaves the metal surface. However, spectroscopy-wise, this leaves the question open as to what is the nature of the linear, ordered structures formed by the dehalogenated precursors. Do those radical species link covalently or via metal-organic coordination, as is commonly the case on $\mathrm{Ag}$ at temperatures below $\sim 150{ }^{\circ} \mathrm{C}$ ? In the absence of clear spectroscopic fingerprints, the answer will be given based on periodicity analysis along the onedimensional structures, organometallic structures typically having significantly larger periodicities than polymers. ${ }^{40,42,43}$

Density functional theory (DFT) calculations for freestanding structures predict periodicities of $9.52 \AA$ for the metal-organic chain (Figure 5c), $8.21 \AA$ for the polymer
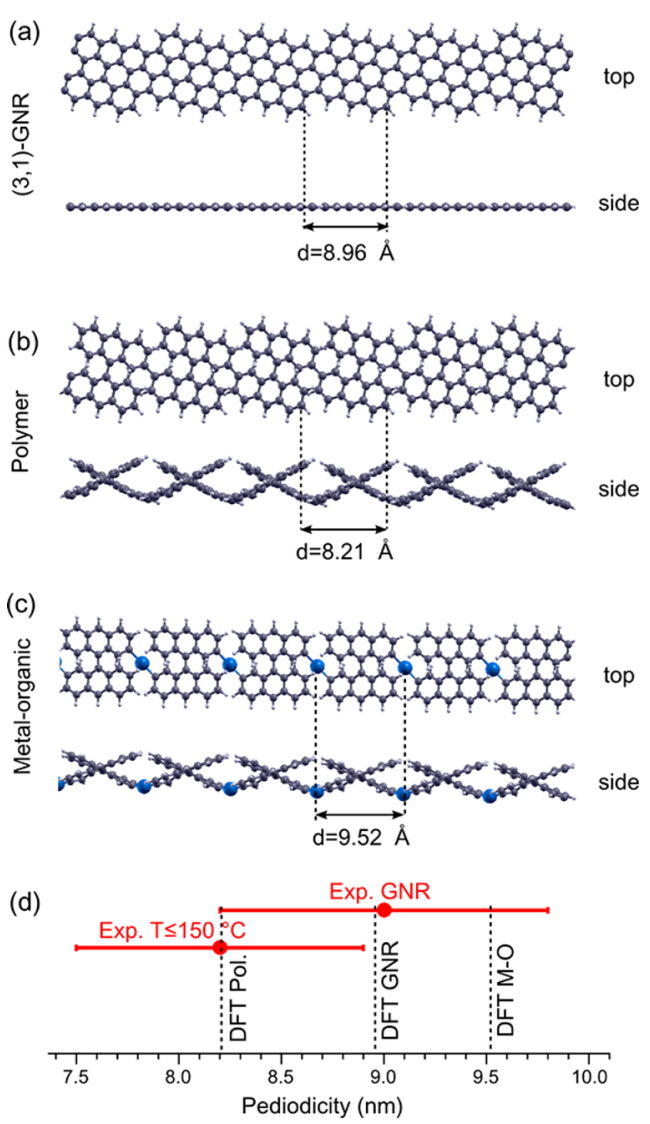

Figure 5. Relaxed structures for (a) free-standing (3,1)-GNRs, (b) poly-2, and (c) metal-organic chains. (d) Comparison of the periodicities of the calculated structures with those measured experimentally for the GNRs and for the nonplanar structure on $\operatorname{Ag}(111)$. The latter fits the polymer period, and its error margin (shown as the standard deviation) is well below the periodicity of the metal-organic chain.

(Figure 5b), and $8.96 \AA$ for the flat (3,1)-GNR (Figure 5a), the latter in good agreement with the value of $8.89 \AA$ that results from assuming an undisturbed graphene lattice for the GNR. Our STM measurements reveal the periodicity of the GNRs to be $9.0 \pm 0.8 \AA$, excellently fitting the calculations. For the nonplanar structures, we observe the same periodicity at room temperature and after annealing to $150{ }^{\circ} \mathrm{C}$, with an average value of $8.2 \pm 0.7 \AA$. The value is similar to that of poly-2 on
$\operatorname{Au}(111)(8.3 \pm 0.6 \AA)$ and in excellent agreement with a polymeric phase, with the error margins remaining clearly under the metal-organic periodicity (Figure $5 \mathrm{~d}$ ). Thus, in spite of the flexibility associated with the nonplanarity of poly-2 and the metal-organic chain, which may introduce minor uncertainties in the calculated periodicities, the results still allow us to conclude that the polymer is directly formed after dehalogenation.

As only reported with a few other systems, ${ }^{44}$ we thus observe a covalent polymer formation readily at room temperature instead of the metal-organic intermediates reported with most precursors linking through Ullmann coupling on $\operatorname{Ag}(111)$ and $\mathrm{Cu}(111) .^{37,39-42}$ The reason behind this may be sought in the different coordination geometry imposed by the nonplanar precursors. As observed in most previous Ullmann coupling studies on $\mathrm{Ag}$ surfaces, $\mathrm{Ag}$ atoms present linear coordination geometry, ${ }^{39,40,42}$ while the anthracene units hosting the generated radicals upon dehalogenation of $\mathbf{2}$ display a strong tilt with respect to the substrate plane due to the steric congestion within the organic backbone (Figure 5c). Under these circumstances, and based on our experimental observations, we conclude that the metal-organic intermediate is not sufficiently stable and the reaction directly proceeds toward the polymeric phase.

As noted above, at $205{ }^{\circ} \mathrm{C}$, the GNRs are fully cyclodehydrogenated on both $\mathrm{Ag}(111)$ and $\mathrm{Au}(111)$. Similarly, low cyclodehydrogenation temperatures $\left(177^{\circ} \mathrm{C}\right)$ are reported for reactant 1 on $\operatorname{Ag}(111),{ }^{28}$ but higher temperatures, similar to those required on $\mathrm{Au}(111)$, are needed to really form GNRs $\left(377^{\circ} \mathrm{C}\right) \cdot{ }^{20,28} \mathrm{On} \mathrm{Cu}(111)$, the required temperature for fully cyclodehydrogenated GNRs to be formed is $250{ }^{\circ} \mathrm{C} .{ }^{33,35}$ Most remarkably, reactant 2 renders fully dehydrogenated GNRs at significantly lower temperatures than $\mathbf{1}$ even on the low reactivity $\mathrm{Au}(111)$ surface. This surprisingly large change relates to the substantially altered strain in the two polymer structures since sterically induced strain is known to weaken the involved $\mathrm{C}-\mathrm{H}$ bonds and thereby lower the cyclodehydrogenation barriers. ${ }^{25,45}$ In poly-1, the anthracene units are linked covalently along their short axis by a bond that allows free rotational movement with respect to their neighbors. This freedom results in alternatively tilted anthracene units along the polymer backbone so as to minimize the steric hindrance from opposing $\mathrm{H}$ atoms. Instead, the anthracene units within poly-2 are linked covalently to their neighbors both along their long and short axes. Thus, although the anthracene units still display the same alternative tilt to reduce the steric hindrance, the covalent bonds along the long anthracene's axes limit the structure's rotational freedom, resulting in a substantially strained geometry. It is this strain opposing the anthracene's tilting which favors the planarization of the structure and thus reduces the cyclodehydrogenation temperature threshold regardless of the substrate.

Both on $\mathrm{Ag}(111)$ and $\mathrm{Au}(111)$, the cyclodehydrogenation threshold is between $150{ }^{\circ} \mathrm{C}$, at which no cyclodehydrogenation is observed, and $205{ }^{\circ} \mathrm{C}$, at which the whole sample has readily become fully planar (Figure 3). On $\operatorname{Ag}(111)$, where polymerization readily starts at room temperature, there is still a substantial temperature gap before the onset of cyclodehydrogenation. However, on $\mathrm{Au}(111)$, the threshold temperatures for polymerization $\left(\sim 125{ }^{\circ} \mathrm{C}\right)$ and cyclodehydrogenation are in close proximity. This may have an impact on the growth process and the resulting GNRs since liberated $\mathrm{H}$ in the cyclodehydrogenation process could quench the available 
radicals and terminate the polymerization. To shed light on this issue, we have increased the number of sampling temperatures, figuring the cyclodehydrogenation onset on $\mathrm{Au}(111)$ to be below $175^{\circ} \mathrm{C}$, at which most of the sample has readily become a planar GNR but some of the polymer units still remain unreacted (inset in Figure 6). A systematic GNR length analysis

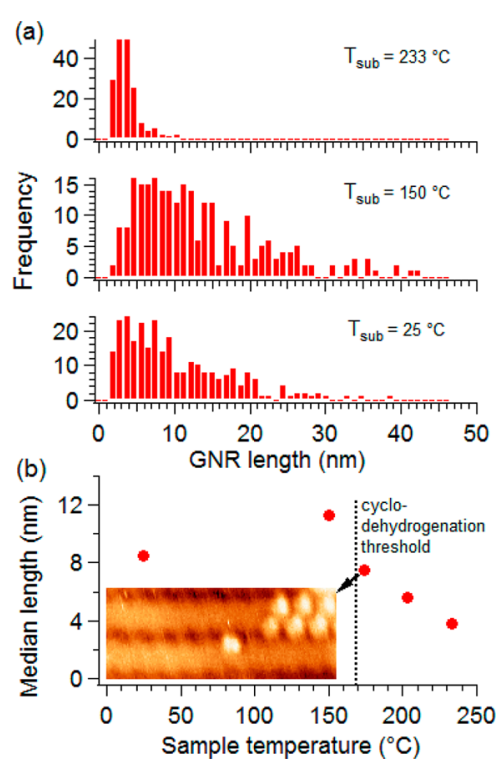

Figure 6. (a) Length distribution of GNRs grown on Au, at coverages close to the full monolayer, for different substrate temperatures upon first deposition. For temperatures below the cyclodehydrogenation threshold $\left(T<174{ }^{\circ} \mathrm{C}\right)$, a second annealing step to $205{ }^{\circ} \mathrm{C}$ was applied to the sample for GNR formation by cyclodehydrogenation. (b) Median length for each substrate temperature. The inset depicts an STM image $(7.4 \mathrm{~nm} \times 2.9 \mathrm{~nm}$, $I=0.16 \mathrm{nA}, U=0.47 \mathrm{~V})$ of a sample deposited at $174{ }^{\circ} \mathrm{C}$, revealing a mostly, but not yet fully, cyclodehydrogenated structure. This value has been thus taken as the cyclodehydrogenation threshold temperature.

of samples as a function of the substrate temperature upon reactant deposition is shown in Figure 6, with all samples having coverages of around 0.8 ML. For temperatures below the cyclodehydrogenation threshold, a second annealing step to $205{ }^{\circ} \mathrm{C}$ was applied to the sample for GNR formation before performing the length analysis.

Representative distributions for selected temperatures are shown in Figure 6a, making immediately obvious that high temperatures narrow the distribution significantly and prevent formation of long GNRs. Because of the asymmetric length distribution, we take the median length as a representative value and plot it versus substrate temperature upon first deposition (Figure 6b). We observe an important drop in the length with increasing temperature once the cyclodehydrogenation threshold is passed. Under this scenario, radical step growth and cyclodehydrogenation take place simultaneously. Thus, radical quenching by liberated $\mathrm{H}$ atoms competes with the radical stepgrowth polymerization. Deposition on $\mathrm{Au}$ held at room temperature and subsequent annealing to cyclodehodrogenation temperatures suffers from the same effect because the precursors on the surface remain intact at room temperature, and both polymerization and cyclodehydrogenation occur during the same subsequent annealing process. However, the length-limiting effect is less pronounced, among other reasons, due to the finite heating rate. Longest GNRs are obtained at substrate temperatures that first activate polymerization, only to form the GNRs in a subsequent annealing process. Under these circumstances, GNRs in excess of $30 \mathrm{~nm}$ can be easily obtained, well beyond the longest (3,1)-GNRs obtained from 1 on $\mathrm{Cu}(111){ }^{30}$ Moreover, additional studies to maximize GNR lengths by optimizing surface coverages or heating rates may bring about even further improvements in the future.

Lastly, we have confirmed the suitability of this molecule to form longer (3,1)-GNRs than $\mathbf{1}$ also on $\mathrm{Cu}(111)$. This is shown in Figure 7 and underlines the great advancement
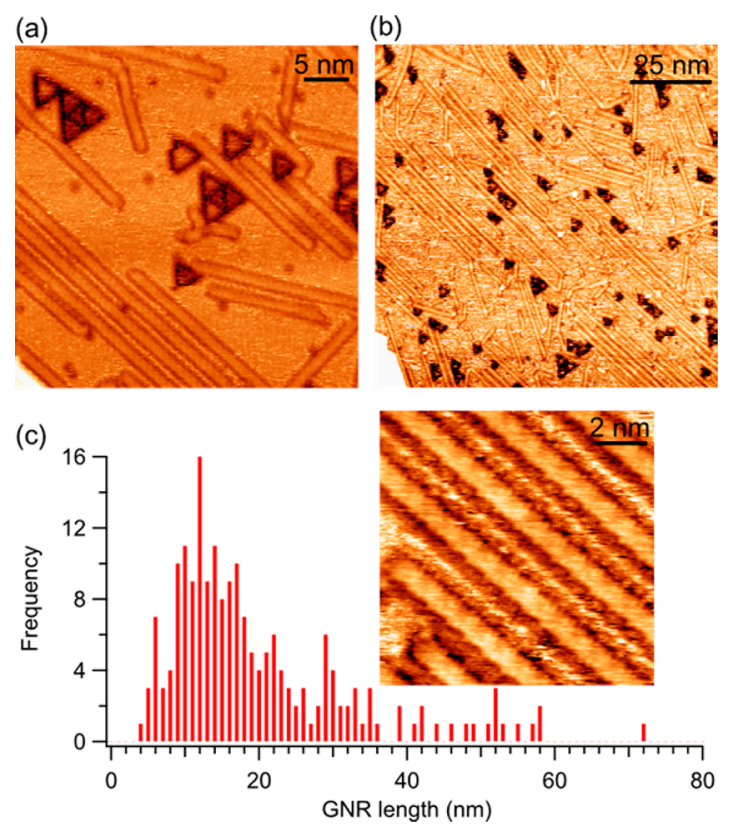

Figure 7. Constant current (a) $36 \times 36 \mathrm{~nm}^{2}(I=0.58 \mathrm{nA}, U=-400$ $\mathrm{mV})$ and (b) $100 \times 100 \mathrm{~nm}^{2}(I=0.05 \mathrm{nA}, U=-200 \mathrm{mV})$ STM images of $(3,1)$-GNRs on $\mathrm{Cu}(111)$. (c) GNR length distribution as obtained from large-scale images as in (b), showing a significant portion of GNRs to be longer than $40-50 \mathrm{~nm}$ and the average and median length values well above those of GNRs grown from 1 on $\mathrm{Cu}(111)$. The inset depicts a $10 \times 10 \mathrm{~nm}^{2}$ close-up view of the GNRs $(I=1 \mathrm{nA}, U=-150 \mathrm{mV})$.

provided by this new GNR precursor. In addition to the $(3,1)$ GNR formation, a concomitant etching of triangular holes into the remaining uncovered $\mathrm{Cu}(111)$ surface is observed, lined along their sides by $\mathrm{Br}$ atoms (Figure 7). A detailed study and description of this process, however, is beyond the scope of this paper. We also want to note that the GNR length analysis of this sample on $\mathrm{Cu}(111)$ (Figure 7c) should not be compared with that on $\mathrm{Au}(111)$ (Figure 6) because the growth was performed in a different chamber with different coverage and a different heating rate, two parameters that may play an important role in the length distribution. However, most importantly, we want to remark that, different from what occurs with precursor 1 on $\mathrm{Cu}(111)$, the precursor 2 allows on the one hand to grow chiral GNRs on different materials not relying on specific and strong molecule-substrate interactions. On the other hand, on all surfaces studied, it forms long GNRs that easily exceed several tens of nanometers, a great advantage for their implementation in actual device structures. 


\section{CONCLUSIONS}

Inspired by the previously reported system-specific growth of $(3,1)$-GNRs on $\mathrm{Cu}(111)$ from precursor 1 , we have designed an alternative building block 2 that renders the same $(3,1)$ GNRs but now independently of the substrate material. This has been proven on $\mathrm{Au}(111), \operatorname{Ag}(111)$, and $\mathrm{Cu}(111)$, revealing additional advantages of the use of this monomer in the growth of selective and atomically precise GNRs, as is the substantially increased length of the resultant GNRs and the low processing temperature required for their formation. Furthermore, the growth process has been followed in detail combining corelevel spectroscopy, scanning tunneling microscopy, and density functional theory calculations, providing a clear correlation between the spectroscopic fingerprints and the different reaction processes, as well as revealing the unusual absence of a metastable metal-organic intermediate preceding the covalent polymerization in the Ullmann coupling process on $\operatorname{Ag}(111)$.

\section{METHODS}

The various metal (111) surfaces were all prepared by standard sputtering-annealing cycles. Subsequently, the samples were prepared by thermal evaporation of 2 at $\sim 140{ }^{\circ} \mathrm{C}$ onto the substrate. Substrate temperature was controlled by resistive heating, and the calibration for the STM experiments was performed by direct measurement of the substrate surface temperature, as a function of the resistive heating current, with a thermocouple fixed and glued to the surface with silver paint after completion of the experiments (see Supporting Information for more details). STM was measured in a commercial UHV system at room temperature, except for the GNR image in Figure 1b, measured in a commercial UHV system at $5 \mathrm{~K}$. WSxM software was used to process all STM images. ${ }^{46}$ XPS measurements were performed using a non-monochromatized source. The XPS data were collected by means of a SPECS Phoibos 100 hemispherical electron analyzer, making use of $\mathrm{Al} \mathrm{K} \alpha \mathrm{X}$-ray emission.

$A b$ initio calculations were carried out on free-standing structures using DFT, as implemented in the SIESTA code. ${ }^{47-49}$ The optB88$\mathrm{vdW}$ functional, which accounts for nonlocal corrections, was adopted for the exchange and correlation potential. We employed a double- $\zeta$ plus polarization basis set and a mesh cutoff of 300 Ry for the realspace integrations. A variable-cell relaxation of the periodic systems was performed until residual forces on all atoms were less than 0.01 $\mathrm{eV} / \AA \AA$ and a Monkhorst-Pack mesh with $101 \times 1 \times 1 k$-point sampling of the three-dimensional Brillouin zone was used.

Details on the four-step synthetic route to obtain dibromobianthracene 2 from phthalic anhydride 3 are given in the Supporting Information.

\section{ASSOCIATED CONTENT}

\section{S Supporting Information}

The Supporting Information is available free of charge on the ACS Publications website at DOI: 10.1021/acsnano.6b05269.

Details on the reactant synthesis, intramolecular resolution STM imaging, description of the temperature measurement method and its calibration, details on the temperature-dependent STM contrast along linear structures (PDF)

\section{AUTHOR INFORMATION}

\section{Corresponding Authors}

*E-mail: d_g_oteyza@ehu.es.

*E-mail: diego.pena@usc.es.

\section{Notes}

The authors declare no competing financial interest.

\section{ACKNOWLEDGMENTS}

The project leading to this publication has received funding from the European Research Council (ERC) under the European Union's Horizon 2020 research and innovation program (Grant Agreement No. 635919), from ICT-FET EU integrated project PAMS (Agreement No. 610446), from the Spanish Ministry of Science and Competitiveness (MINECO, Grant Nos. MAT2013-46593-C6-01, MAT2013-46593-C6-2-P, MAT2013-46593-C6-4-P, MAT2013-46593-C6-6-P), FEDER, from the Basque Government (Grant Nos. IT-621-13, IT-75613, and PI2015-042), from the World Premier International Center (WPI) for Materials Nanoarchitectonics (MANA) of the National Institute for Materials Science (NIMS), Tsukuba, Japan, and from JSPS Bilateral Open Partnership Joint Research Project.

\section{REFERENCES}

(1) Talirz, L.; Ruffieux, P.; Fasel, R. On-Surface Synthesis of Atomically Precise Graphene Nanoribbons. Adv. Mater. 2016, 28, 6222-6231.

(2) Narita, A.; Wang, X.-Y.; Feng, X.; Müllen, K. New Advances in Nanographene Chemistry. Chem. Soc. Rev. 2015, 44, 6616-6643.

(3) Son, Y.-W.; Cohen, M. L.; Louie, S. G. Energy Gaps in Graphene Nanoribbons. Phys. Rev. Lett. 2006, 97, 216803.

(4) Yang, L.; Park, C.-H.; Son, Y.-W.; Cohen, M. L.; Louie, S. G. Quasiparticle Energies and Band Gaps in Graphene Nanoribbons. Phys. Rev. Lett. 2007, 99, 186801.

(5) Jiang, Z.; Song, Y. Band Gap Oscillation and Novel Transport Property in Ultrathin Chiral Graphene Nanoribbons. Phys. B 2015, 464, 61-67.

(6) Yazyev, O. V.; Capaz, R. B.; Louie, S. G. Theory of Magnetic Edge States in Chiral Graphene Nanoribbons. Phys. Rev. B: Condens. Matter Mater. Phys. 2011, 84, 115406.

(7) Tao, C.; Jiao, L.; Yazyev, O. V.; Chen, Y.-C.; Feng, J.; Zhang, X.; Capaz, R. B.; Tour, J. M.; Zettl, A.; Louie, S. G.; Dai, H.; Crommie, M. F. Spatially Resolving Edge States of Chiral Graphene Nanoribbons. Nat. Phys. 2011, 7, 616-620.

(8) Cloke, R. R.; Marangoni, T.; Nguyen, G. D.; Joshi, T.; Rizzo, D. J.; Bronner, C.; Cao, T.; Louie, S. G.; Crommie, M. F.; Fischer, F. R. Site-Specific Substitutional Boron Doping of Semiconducting Armchair Graphene Nanoribbons. J. Am. Chem. Soc. 2015, 137, 88728875.

(9) Kawai, S.; Saito, S.; Osumi, S.; Yamaguchi, S.; Foster, A. S.; Spijker, P.; Meyer, E. Atomically Controlled Substitutional BoronDoping of Graphene Nanoribbons. Nat. Commun. 2015, 6, 8098.

(10) Nguyen, G. D.; Toma, F. M.; Cao, T.; Pedramrazi, Z.; Chen, C.; Rizzo, D. J.; Joshi, T.; Bronner, C.; Chen, Y.-C.; Favaro, M.; Louie, S. G.; Fischer, F. R.; Crommie, M. F. Bottom-Up Synthesis of N = 13 Sulfur-Doped Graphene Nanoribbons. J. Phys. Chem. C 2016, 120, 2684-2687.

(11) Basagni, A.; Vasseur, G.; Pignedoli, C. A.; Vilas-Varela, M.; Peña, D.; Nicolas, L.; Vitali, L.; Lobo-Checa, J.; de Oteyza, D. G.; Sedona, F.; Casarin, M.; Ortega, J. E.; Sambi, M. Tunable Band Alignment with Unperturbed Carrier Mobility of On-Surface Synthesized Organic Semiconducting Wires. ACS Nano 2016, 10, 2644-2651.

(12) Cai, J.; Pignedoli, C. A.; Talirz, L.; Ruffieux, P.; Söde, H.; Liang, L.; Meunier, V.; Berger, R.; Li, R.; Feng, X.; Müllen, K.; Fasel, R. Graphene Nanoribbon Heterojunctions. Nat. Nanotechnol. 2014, 9, 896-900.

(13) Bronner, C.; Stremlau, S.; Gille, M.; Brauße, F.; Haase, A.; Hecht, S.; Tegeder, P. Aligning the Band Gap of Graphene Nanoribbons by Monomer Doping. Angew. Chem., Int. Ed. 2013, 52, $4422-4425$.

(14) Zhang, X.; Yazyev, O. V.; Feng, J.; Xie, L.; Tao, C.; Chen, Y.-C.; Jiao, L.; Pedramrazi, Z.; Zettl, A.; Louie, S. G.; Dai, H.; Crommie, M. F. Experimentally Engineering the Edge Termination of Graphene Nanoribbons. ACS Nano 2013, 7, 198-202. 
(15) Chen, Y.-C.; Cao, T.; Chen, C.; Pedramrazi, Z.; Haberer, D.; de Oteyza, D. G.; Fischer, F.; Louie, S. G.; Crommie, M. F. Molecular Bandgap Engineering of Bottom-Up Synthesized Graphene Nanoribbon Heterojunctions. Nat. Nanotechnol. 2015, 10, 156-160.

(16) Sevinçli, H.; Topsakal, M.; Ciraci, S. Superlattice Structures of Graphene-Based Armchair Nanoribbons. Phys. Rev. B: Condens. Matter Mater. Phys. 2008, 78, 245402.

(17) Prezzi, D.; Varsano, D.; Ruini, A.; Molinari, E. Quantum Dot States and Optical Excitations of Edge-Modulated Graphene Nanoribbons. Phys. Rev. B: Condens. Matter Mater. Phys. 2011, 84, 041401.

(18) Schwierz, F. Graphene Transistors. Nat. Nanotechnol. 2010, 5, 487-496.

(19) Bennett, P. B.; Pedramrazi, Z.; Madani, A.; Chen, Y.-C.; de Oteyza, D. G.; Chen, C.; Fischer, F. R.; Crommie, M. F.; Bokor, J. Bottom-Up Graphene Nanoribbon Field-Effect Transistors. Appl. Phys. Lett. 2013, 103, 253114.

(20) Cai, J.; Ruffieux, P.; Jaafar, R.; Bieri, M.; Braun, T.; Blankenburg, S.; Muoth, M.; Seitsonen, A. P.; Saleh, M.; Feng, X.; Mullen, K.; Fasel, R. Atomically Precise Bottom-up Fabrication of Graphene Nanoribbons. Nature 2010, 466, 470-473.

(21) Chen, Y.-C.; de Oteyza, D. G.; Pedramrazi, Z.; Chen, C.; Fischer, F. R.; Crommie, M. F. Tuning the Band Gap of Graphene Nanoribbons Synthesized from Molecular Precursors. ACS Nano 2013, 7, 6123-6128.

(22) Zhang, H.; Lin, H.; Sun, K.; Chen, L.; Zagranyarski, Y.; Aghdassi, N.; Duhm, S.; Li, Q.; Zhong, D.; Li, Y.; Mullen, K.; Fuchs, H.; Chi, L. On-Surface Synthesis of Rylene-Type Graphene Nanoribbons. J. Am. Chem. Soc. 2015, 137, 4022-4025.

(23) Kimouche, A.; Ervasti, M. M.; Drost, R.; Halonen, S.; Harju, A.; Joensuu, P. M.; Sainio, J.; Liljeroth, P. Ultra-Narrow Metallic Armchair Graphene Nanoribbons. Nat. Commun. 2015, 6, 10177.

(24) Ruffieux, P.; Wang, S.; Yang, B.; Sánchez-Sánchez, C.; Liu, J.; Dienel, T.; Talirz, L.; Shinde, P.; Pignedoli, C. A.; Passerone, D.; Dumslaff, T.; Feng, X.; Müllen, K.; Fasel, R. On-Surface Synthesis of Graphene Nanoribbons with Zigzag Edge Topology. Nature 2016, 531, 489-492.

(25) Blankenburg, S.; Cai, J.; Ruffieux, P.; Jaafar, R.; Passerone, D.; Feng, X.; Mullen, K.; Fasel, R.; Pignedoli, C. A. Intraribbon Heterojunction Formation in Ultranarrow Graphene Nanoribbons. ACS Nano 2012, 6, 2020-2025.

(26) Bjork, J.; Stafstrom, S.; Hanke, F. Zipping Up: Cooperativity Drives the Synthesis of Graphene Nanoribbons. J. Am. Chem. Soc. 2011, 133, 14884-14887.

(27) Batra, A.; Cvetko, D.; Kladnik, G.; Adak, O.; Cardoso, C.; Ferretti, A.; Prezzi, D.; Molinari, E.; Morgante, A.; Venkataraman, L. Probing the Mechanism for Graphene Nanoribbon Formation on Gold Surfaces Through X-Ray Spectroscopy. Chem. Sci. 2014, 5, $4419-4423$

(28) Huang, H.; Wei, D.; Sun, J.; Wong, S. L.; Feng, Y. P.; Castro Neto, A. H.; Wee, A. T. S. Spatially Resolved Electronic Structures of Atomically Precise Armchair Graphene Nanoribbons. Sci. Rep. 2012, 2, 983.

(29) Han, P.; Akagi, K.; Canova, F. F.; Mutoh, H.; Shiraki, S.; Iwaya, K.; Weiss, P. S.; Asao, N.; Hitosugi, T. Bottom-Up GrapheneNanoribbon Fabrication Reveals Chiral Edges and Enantioselectivity. ACS Nano 2014, 8, 9181-9187.

(30) Han, P.; Akagi, K.; Canova, F. F.; Shimizu, R.; Oguchi, H.; Shiraki, S.; Weiss, P. S.; Asao, N.; Hitosugi, T. Self-Assembly Strategy for Fabricating Connected Graphene Nanoribbons. ACS Nano 2015, 9, 12035-12044.

(31) Han, P.; Akagi, K.; Canova, F. F.; Mutoh, H.; Shiraki, S.; Iwaya, K.; Weiss, P. S.; Asao, N.; Hitosugi, T. Reply to "Comment on 'Bottom-Up Graphene-Nanoribbon Fabrication Reveals Chiral Edges and Enantioselectivity'. ACS Nano 2015, 9, 3404-3405.

(32) Simonov, K. A.; Vinogradov, N. A.; Vinogradov, A. S.; Generalov, A. V.; Zagrebina, E. M.; Martensson, N.; Cafolla, A. A.; Carpy, T.; Cunniffe, J. P.; Preobrajenski, A. B. Effect of Substrate Chemistry on the Bottom-Up Fabrication of Graphene Nanoribbons:
Combined Core-Level Spectroscopy and STM Study. J. Phys. Chem. C 2014, 118, 12532-12540.

(33) Simonov, K. A.; Vinogradov, N. A.; Vinogradov, A. S.; Generalov, A. V.; Zagrebina, E. M.; Martensson, N.; Cafolla, A. A.; Carpy, T.; Cunniffe, J. P.; Preobrajenski, A. B. Comment on "BottomUp Graphene-Nanoribbon Fabrication Reveals Chiral Edges and Enantioselectivity. ACS Nano 2015, 9, 3399-3403.

(34) Simonov, K. A.; Vinogradov, N. A.; Vinogradov, A. S.; Generalov, A. V.; Zagrebina, E. M.; Svirskiy, G. I.; Cafolla, A. A.; Carpy, T.; Cunniffe, J. P.; Taketsugu, T.; Lyalin, A.; Martensson, N.; Preobrajenski, A. B. From Graphene Nanoribbons on $\mathrm{Cu}(111)$ to Nanographene on $\mathrm{Cu}(110)$ : Critical Role of Substrate Structure in the Bottom-Up Fabrication Strategy. ACS Nano 2015, 9, 8997-9011.

(35) Sánchez-Sánchez, C.; Dienel, T.; Deniz, O.; Ruffieux, P.; Berger, P.; Feng, X.; Müllen, K.; Fasel, R. Purely Armchair or Partially Chiral: Non-Contact Atomic Force Microscope Characterization of DibromoBianthryl-Based Graphene Nanoribbons Grown on $\mathrm{Cu}(111)$. ACS Nano 2016, 10, 8006-8011.

(36) Weiler-Feilchenfeld, H.; Bergmann, E. D.; Hirschfeld, A. The Conformation of 9,9'-bianthryl. Tetrahedron Lett. 1965, 6, 4129-4131.

(37) Chen, M.; Xiao, J.; Steinrück, H.-P.; Wang, S.; Wang, W.; Lin, N.; Hieringer, W.; Gottfried, J. M. Combined Photoemission and Scanning Tunneling Microscopy Study of the Surface-Assisted Ullmann Coupling Reaction. J. Phys. Chem. C 2014, 118, 6820-6830.

(38) Basagni, A.; Sedona, F.; Pignedoli, C. A.; Cattelan, M.; Nicolas, L.; Casarin, M.; Sambi, M. Molecules-Oligomers-NanowiresGraphene Nanoribbons: A Bottom-Up Stepwise On-Surface Covalent Synthesis Preserving Long-Range Order. J. Am. Chem. Soc. 2015, 137, $1802-1808$

(39) Dong, L.; Liu, P. N.; Lin, N. Surface-Activated Coupling Reactions Confined on a Surface. Acc. Chem. Res. 2015, 48, 27652774.

(40) Shi, K. J.; Yuan, D. W.; Wang, C. X.; Shu, C. H.; Li, D. Y.; Shi, Z. L.; Wu, X. Y.; Liu, P. N. Ullmann Reaction of Aryl Chlorides on Various Surfaces and the Application in Stepwise Growth of 2D Covalent Organic Frameworks. Org. Lett. 2016, 18, 1282-1285.

(41) Di Giovannantonio, M.; El Garah, M.; Lipton-Duffin, J.; Meunier, V.; Cardenas, L.; Fagot Revurat, Y.; Cossaro, A.; Verdini, A.; Perepichka, D. F.; Rosei, F.; Contini, G. Insight into Organometallic Intermediate and Its Evolution to Covalent Bonding in SurfaceConfined Ullmann Polymerization. ACS Nano 2013, 7, 8190-8198.

(42) Walch, H.; Gutzler, R.; Sirtl, T.; Eder, G.; Lackinger, M. Material- and Orientation-Dependent Reactivity for Heterogeneously Catalyzed Carbon-Bromine Bond Homolysis. J. Phys. Chem. C 2010, $114,12604-12609$

(43) Zhang, Y. Q.; Kepcija, N.; Kleinschrodt, M.; Diller, K.; Fischer, S.; Papageorgiou, A. C.; Allegretti, F.; Bjork, J.; Klyatskaya, S.; Klappenberger, F.; Ruben, M.; Barth, J. V. Homo-Coupling of Terminal Alkynes on a Noble Metal Surface. Nat. Commun. 2012, 3, 1286.

(44) Lahrood, A. R.; Björk, J.; Heckl, W. M.; Lackinger, M. 1,3Diiodobenzene on $\mathrm{Cu}(111)$ - an Exceptional Case of On-Surface Ullmann Coupling. Chem. Commun. 2015, 51, 13301-13304.

(45) Treier, M.; Pignedoli, C. A.; Laino, T.; Rieger, R.; Müllen, K.; Passerone, D.; Fasel, R. Surface-Assisted Cyclodehydrogenation Provides a Synthetic Route towards Easily Processable and Chemically Tailored Nanographenes. Nat. Chem. 2011, 3, 61-67.

(46) Horcas, I.; Fernández, R.; Gómez-Rodríguez, J. M.; Colchero, J.; Gómez-Herrero, J.; Baro, A. M. WSXM: A Software for Scanning Probe Microscopy and a Tool for Nanotechnology. Rev. Sci. Instrum. 2007, 78, 013705.

(47) Soler, J. M.; Artacho, E.; Gale, J. D.; Garcia, A.; Junquera, J.; Ordejón, P.; Sanchez-Portal, D. The SIESTA Method for Ab Initio Order-N Materials Simulation. J. Phys.: Condens. Matter 2002, 14, 2745.

(48) Sánchez-Portal, D.; Ordejón, P.; Artacho, E.; Soler, J. M. Density-Functional Method for Very Large Systems with LCAO Basissets. Int. J. Quantum Chem. 1997, 65, 453-461. 
(49) Artacho, E.; Sánchez-Portal, D.; Ordejón, P.; Garcia, A.; Soler, J. M. Linear-Scaling Ab-Initio Calculations for Large and Complex Systems. Phys. Status Solidi B 1999, 215, 809-817. 